Gregor PERKO

Université de Ljubljana

\title{
LE MÉTALINGUISTIQUE ET LE PÉRIPHÉRIQUE EN MORPHOLOGIE CONSTRUCTIONNELLE
}

\section{Introduction}

Le présent article vise à étudier le rapport entre la dimension métalinguistique de certains procédés constructionnels et de certaines unités complexes et le fait que ces procédés et ces unités se situent à la périphérie (ou en-dehors) du système grammatical du français. Cette relation entre le périphérique et le métalinguistique dans le domaine de la morphologie constructionnelle ne saurait surprendre puisque tout emploi métalinguistique du langage est considéré comme un emploi non prototypique ou périphérique du langage (cf. REY-DEBOVE, 1997).

La formation des unités lexicales en français ne se réduit pas à la dérivation et à la composition que la plupart des modèles morphologiques contemporains (approches structuralistes ou génératives, modèle Silex, théorie »Sens-Texte«, morphologie naturelle, entre autres) considèrent, implicitement ou explicitement, ${ }^{1}$ comme des procédés centraux partageant certaines caractéristiques prototypiques : les deux procédés emploient des moyens grammaticaux, morphologiques ou syntaxiques, réguliers, ils sont utilisés à des fins dénominatives, le locuteur qui les met en oeuvre ne le fait pas d'une manière consciente (cf. FRADIN, 2003 : 207208). Une partie des unités lexicales est produite par des moyens dits périphériques, non grammaticaux, extragrammaticaux ou marginaux. ${ }^{2}$

Les critères sur lesquels s'appuie la distinction entre procédés centraux et périphériques sont assez hétérogènes. Pour définir les procédés périphériques (extragrammaticaux), la littérature contemporaine (DOLESCHAL \& THORNTON (éds.), 2000 ; FRADIN, 2003 ; DRESSLER \& KASTOVSKY \& PFEIFFER \& RAINER 2005) met en avant principalement les critères suivants :

a) absence de contraintes (catégorielles, sémantiques) qui pèsent sur la sélection des bases ;

b) changement sémantique faible ;

\footnotetext{
${ }^{1}$ La place privilégiée qu'occupent ces deux types de formation se manifeste dans le fait que la plupart des monographies et des manuels consacrés à la morphologie constructionnelle ne s'intéressent qu'à ces deux types de formation et laissent de côté les autres procédés de formation des mots. Le Cours de morphologie générale de I. A. Mel'čuk en est un bon exemple : «[l]a formation synchronique de mots est constituée de deux composantes: la dérivation et la composition.» (MEL'CUK, 1993 : 317).

Il convient d'ajouter que les théories morphologiques établissent également une hiérarchie entre les deux types «centraux » et même à l'intérieur de chacun des deux types : la dérivation est un procédé constructionnel plus «central » ou plus «prototypique » que la composition (cf. CoRBIN, 1997 ; DRESSLER, 2005) ; la suffixation est plus «importante » que la préfixation (MEL'ČUK, 1997 : 151 ; cf. CORBIN, 2001).

${ }^{2}$ Pour les besoins de notre étude, nous ne différencierons pas les termes cités, ce qui, bien entendu, n'est pas le cas de tous les cadres théoriques. W. U. Dressler, par exemple, distingue nettement entre morphologie extragrammaticale et morphologie marginale, mais reconnaît lui-même que cette distinction repose sur les prémisses théoriques spécifiques («theory-dependent ») de la morphologie naturelle (DRESSLER, $2000: 1$ ).
} 
c) ces procédés n'obéissent pas à des patrons réguliers et ne sont donc pas productifs ${ }^{3}$;

d) l'application de ces procédés est intentionnelle ou consciente ;

e) les unités issues de ces procédés sont marquées ${ }^{4}$;

f) ces procédés ne sont pas descriptifs et ne répondent pas à des besoins de dénomination, mais relèvent du registre de l'affectif ou du ludique.

Les procédés morphologiques les plus fréquemment évoqués comme périphériques (extragrammaticaux) (cf. DOLESCHAL \& THORNTON (éds.), 2000 ; FRADIN, 2003 : 206-220) sont: formation de mots-échos et de mots-valises, abréviation, troncation, siglaison, verlanisation, suffixation parasitaire, suffixation sécrétive, composition cachée, composition savante et dérivation évaluative. Comme nous le verrons plus loin ( $\$$ 2.2.), tous les procédés énumérés ne remplissent pas tous les critères cités.

L'étude proposée dans cet article, conjuguant la morphologie constructionnelle avec la sémiotique, ne s'inscrit pas dans un cadre théorique précis. Une légère préférence sera toutefois accordée à la morphologie naturelle (cf. DRESSLER \& MAYERTHALER \& PANAGL \& WURZEL, 1987) pour la simple raison que c'est au sein et dans les parages de cette théorie qu'on trouve les recherches les plus intéressantes sur la morphologie «périphérique ».

\section{Remarque préliminaire}

Lorsque nous parlons du caractère métalinguistique de procédés constructionnels et d'unités complexes, nous pensons à deux phénomènes différents mais étroitement imbriqués :

- d'un côté, il s'agit de procédés qui exploitent de façon imprédictible et irrégulière le plan du signifiant, ce qui veut dire qu'il n'y a pas de rapport direct ou régulier entre le plan du signifiant et le plan du signifié : c'est le cas par exemple des mots-échos (glouglou), des troncations (manif $\leftarrow$ manifestation), des verlanisations (askeum $\leftarrow$ comme aç $\leftarrow$ comme ça) ou des mots-valises (castring $\leftarrow$ casting en string);

- de l'autre côté, nous nous intéresserons à la connotation autonymique (REY-DEBOVE, 1997: 251-291) qui désigne un statut de l'unité lexicale complexe intermédiaire entre l'emploi ordinaire et l'emploi autonymique dans le sens où une unité complexe réfère au monde mais, en même temps, connote sa propre structure morphologique; par exemple, un néologisme comme roule-couilles, qui désigne un slip, un string, «frappe » sans doute par sa structure morphologique : V+N, composé à partir de rouler les couilles, par analogie avec rouler les mécaniques (SABLAYROLLES, 1993 : 226).

\footnotetext{
${ }^{3}$ Sur les difficultés que pose en morphologie constructionnelle la notion de productivité, voir DAL (2003).

${ }^{4}$ Le trait [ \pm marqué] constitue un des fondements de l'approche naturelle (cf. DRESSLER \& MAYerthaler \& PANAgl \& Wurzel, 1987). Nous le remplacerons par le concept, plus précis, de connotation autonymique (voir $\S 3$ ).
} 


\section{Les procédés à caractère métalinguistique}

\subsection{Typologie des procédés}

Force est de constater que la majeure partie des procédés de formation des mots que les approches théoriques contemporaines situent à la périphérie ou en dehors du système grammatical présentent un caractère métalinguistique. ${ }^{5}$ Ces procédés sont extrêmement variés. Pour les besoins de notre propos nous les regrouperons en trois types.

a) Les procédés qui présentent un certain degré d'iconicité, c'est-à-dire que les unités produites par ces procédés possèdent des propriétés de leurs dénotata (ECO, 1988 : 80). Ce sont par exemple les mots-échos (ang. echo-words, MORIN, 1972) dont une partie non négligeable est de formation onomatopéique (tic tac, glouglou, gnangnan, guili-guili, ronron, teuf teuf, zig zag). ${ }^{6}$

b) Les procédés d'abréviation, de transposition ou d'ajout qui ne font pas intervenir des changements sémantiques notables, comme la troncation (manif, ordi), la siglaison (SMIC, RMI, CGT), la verlanisation ${ }^{7}$ (tromé, meuf, iech), la suffixation parasitaire (bombe $\rightarrow$ bombax).

c) Les procédés se rapprochant de la dérivation ou de la composition, mais dans lesquels, au moins à l'origine, le patron phonologique et le patron sémantique ne concordent pas. Dans cette catégorie nous pouvons ranger :

- la formation de mots-valises (GRÉSILLON, 1984) : clavardage, courriel...

- l'affixation sécrétive (ang. secretade affixing ; FRADIN, $2000: 35-47)^{8}$ : [pére]stroïka $\rightarrow$ castroïka, sextrö̈ka....

- la composition cachée (ang. concealed compounding ; FRADIN, 2000 : 47-52) $)^{9}$ : [logi] ciel $\rightarrow$ didacticiel, ludiciel, orthociel...

Ce qui est commun à tous ces procédés, c'est qu'au moins un des éléments entrant dans les trois types de formation ne peut pas être considéré comme un morphème lexical (cla[vier] [ba]vardage ; -strö̈ka, -ciel). La différence principale entre les trois types semble concerner la construction du sens du lexème complexe :

- la construction du sens d'un mot-valise est identique à la construction du sens des composés ;

- l'affixe sécrété n'apporte qu'une partie du sens du lexème dont il est dérivé : tous les néologismes en - stroïka désignent un renouveau sans pourtant référer aux conditions politiques, sociales et historiques de l'URSS des années Gorbatchev ;

\footnotetext{
${ }^{5}$ Parmi les procédés périphériques ne présentant pas de caractère métalinguistique, mentionnons notamment la composition savante (FRADIN, 2003 : 197-199) et la dérivation évaluative (DRESSLER \& MERLINI BARBARESI, 1994). Nous y reviendrons au $§ 2.3$.

${ }^{6} \mathrm{Si}$ tous les mots-échos ne sont pas des onomatopées (coco, nana, neuneu...), la frontière entre les mots-échos onomatopéiques et non onomatopéiques est cependant difficile à tracer (dare-dare).

${ }^{7}$ On pourrait y ajouter encore d'autres procédés argotiques «cryptiques » comme le javanais ou le loucherbem.

${ }^{8}$ L'un des formants est un lexème (ou une abréviation), l'autre un «affixe sécrété » qui véhicule une partie du contenu sémantique du lexème dont il est « extrait ».

${ }^{9}$ L'un des formants est un lexème (ou une abréviation), l'autre un lexème abrégé qui véhicule l'intégralité du contenu sémantique du lexème de base et assure la cohérence du paradigme.
} 
- dans la composition cachée, l'élément donnant une cohérence au paradigme apporte l'intégralité du sens du lexème de base : tous les composés en ciel désignent des logiciels.

\subsection{Hétérogénéité des procédés et insuffisance des critères énumérés}

Nous avons déjà fait remarquer (voir Introduction ci-dessus) que la distinction entre procédés centraux et périphériques reposait sur des critères hétérogènes. Étant donné que la catégorie de la morphologie constructionnelle périphérique est une catégorie à la fois hétérogène et prototypique, il est clair que tous les procédés ne satisfont pas à tous les critères énumérés dans l'introduction de cet article, à savoir : a) bases non contraintes, b) changement sémantique faible, c) caractère irrégulier, non productif ; d) caractère intentionnel ; e) caractère marqué ; f) registre affectif, non descriptif. Les procédés présentant un caractère métalinguistique «fondamental » et qui sont regroupés sous a) et b) ci-dessus (§2.1.), à savoir la formation des mots-échos, la troncation, la siglaison, la verlanisation et la suffixation parasitaire, satisfont à tous ou à presque tous les critères. Par conséquent, ces procédés doivent être considérés comme des procédés périphériques prototypiques et donc comme les procédés les plus éloignés du centre englobant la dérivation et la composition :

- les mots-échos, la verlanisation obéissent à tous les critères évoqués ${ }^{10}$;

- le siglaison se rapproche du centre par son caractère descriptif ;

- la troncation impose des contraintes sémantiques, ${ }^{11}$ la suffixation parasitaire des contraintes catégorielles ${ }^{12}$;

- la siglaison ne satisfait pas au critère f).

Parmi les procédés regroupés sous c) (\$2.1.), les mots-échos obéissent à tous les critères à l'exception de b) : la formation de mots-valises implique un changement sémantique important.

L'affixation sécrétive pose plusieurs problèmes mais se situe encore à la périphérie de la morphologie constructionnelle. Dans un premier temps, il faut se demander s'il existe des contraintes qui pèsent sur la sélection des bases. A première vue, la réponse est affirmative : les affixes sécrétés (fr.-stroïka de Péreströ̈ka ou ang. -gate de Watergate) ne choisissent que des bases nominales. Or, cette contrainte n'est pas de nature grammaticale, mais découle tout naturellement du fait que les affixés sécrétifs, au moins dans le corpus réuni, ne désignent que des événements ou des phénomènes, qui sont en français, bien évidemment, dénotés par la catégorie morphologique des noms. Un deuxième problème concerne le caractère descriptif du procédé. Bien que la fonction

\footnotetext{
${ }^{10}$ En morphologie naturelle, les mots d'origine onomatopéique sont considérés comme les mots les plus iconiques et relèvent forcément de la morphologie extragrammaticale : «En particulier les plus iconiques, c'est-à-dire les images, n'apparaissent à strictement parler qu'en morphologie extragrammaticale où les bases universelles ne sont pas restreintes par la normalité du système spécifique » (KILANI-SCHOCH \& DRESSLER, 2005 : 40-41).

${ }^{11}$ En règle générale, la troncation n'est possible qu'avec une partie des acceptions du mot-base. Par exemple, manifestation: 1 . démonstration collective, 2 . expression $\rightarrow$ manif contre la guerre vs. *manif de joie.

${ }^{12}$ Par exemple, -anche est un suffixe nominal (boutanche), -arès un suffixe verbal (bouclarès), -os un suffixe essentiellement adjectival (calmos).
} 
dénominative semble dominer, ces affixés ne peuvent pas se départir d'une dimension affective ou connotative qui est la conséquence du dernier problème que nous évoquerons. En effet, le caractère métalinguistique du procédé se double d'un caractère qu'on pourrait nommer «intertextuel»: tout emploi de l'affixe sécrété renvoie nécessairement au lexème dont il a été sécrété. ${ }^{13}$

Parmi les procédés étudiés au $§ 2.1$, la composition cachée est sans doute celui qui se situe le plus près de la frontière séparant le centre de la périphérie. Il reste à décider de quel côté de la frontière la ranger. Le caractère descriptif évident et le changement sémantique important que ce type de composition implique situent la composition cachée du côté des procédés centraux. Les contraintes catégorielles pesant sur la base ne semblent toutefois pas de nature linguistique. ${ }^{14}$ En raison de la cohérence sémantique du paradigme des formants de ce procédé (ciel dans logiciel $\rightarrow$ didacticiel, imagiciel, ludiciel, orthociel, progiciel ou télé- de téléachat, téléévangéliste, téléfilm ), ces formants doivent être considérés, en synchronie, comme non marqués et réguliers (voir aussi ci-dessous $§ 2.3$ ).

Suivant FRADIN (2000) et CORBIN (2001), ${ }^{15}$ nous avons traité la composition cachée comme un type de procédé morphologique à part. Les approches qui s'appuient sur le concept de confixation de A. MARTINET (1996 : 135) assimilent, en revanche, la composition cachée à la composition savante, ce qui est dans une perspective synchronique sans doute une meilleure solution : le seul critère fiable permettant de distinguer le formant savant télé - («à distance) des formants de la composition cachée télé- (de «télévision»), télé- (de «téléphone») ou télé (de «téléphérique ») est l'étymologie. Dans leur manuel de lexicologie française, F. Gaudin et L. Guespin, par exemple, parlent de deux types de recomposés : recomposés classiques (agronomie, biologie, autodétermination) et recomposés modernes (agroalimentaire, bioéthique, autostoppeur) (GAUDIN § GUESPIN, 2000 : 287-291). Un traitement unifié des deux procédés est également proposé par J. Radimský dans sa monographie sur les composés en italien actuel : il distingue les confixes classiques et les confixes modernes (appelés aussi néoconfixes) (RADIMSKÝ, 2006 : 62-63, 110-112).

\subsection{Pour un critère discriminatif unique}

La confrontation entre les critères distinguant les procédés centraux (ou grammaticaux) des périphériques (ou extragrammaticaux) discutés en 2.2. et le caractère métalinguistique de certains procédés constructionnels ont montré que le caractère métalinguistique permettait à lui seul la distinction entre les procédés périphériques prototypiques et les procédés centraux prototypiques. La zone

\footnotetext{
${ }^{13}$ D'où la différence de connotation entre mongolstroïka (affixé sécrétif) et réforme en Mongolie (syntagme descriptif neutre) (FRADIN, $2003: 212-213$ ).

${ }^{14}$ On peut en donner grosso modo la même explication que celle qu'on a proposée pour l'affixation sécrétive. On tombe, en outre, sur les forums ou les blogs internet, sur des composés comme télacheter ou télévendre, ce qui prouve qu'au moins en principe ces formants ne sont pas uniquement nominaux (téléachat, téléacheteur, télévente, télévendeur...), mais peuvent s'appliquer également aux verbes.

15 Danielle Corbin appelle les formants (unités infralexicales) de la composition cachée des fractoconstituants qu'elle distingue des affixes. Le même statut est réservé aux formants de la composition savante, appelés archéoconstituants (CORBIN, 2001 : 44).
} 
d'ombre qui subsiste et qui couvre la composition cachée, la composition savante et la dérivation évaluative demande quelques clarifications.

Il est indéniable que le statut central ou périphérique n'est pas indépendant des modèles théoriques particuliers. Dans les modèles structuralistes et associatifs, les compositions savante et cachée sont écartées du centre du fait qu'elles utilisent des bases non autonomes sur le plan morphologique, mais autonomes sur le plan sémantique. ${ }^{16}$ La dérivation évaluative est «marginalisée » surtout dans les approches s'inspirant de la morphologie naturelle (DRESSLER \& MERLINI BARBARESI, 1994): les dérivés évaluatifs sont marqués, ils relèvent plus du registre affectif ou ludique que du registre descriptif, les catégories morphologique et sémantique des dérivés ne changent pas.

Le statut plus périphérique que central des trois procédés repose cependant également sur des facteurs plus universels. Les compositions savante et cachée utilisent des formants qui, étymologiquement, ne sont pas des formants français, les uns sont d'origine grecque ou latine, les autres ne sont que des segments plus ou moins « aléatoires » de mots français, n'obéissant qu'à des contraintes phonologiques. Dans une perspective synchronique, ces critères n'entrent plus en jeu :

- les formants «savants» fonctionnent aujourd'hui en français plus ou moins indépendamment de leur origine étrangère ;

- les formants «modernes » de la composition cachée perdent, à force de former des paradigmes cohérents, leur caractère métalinguistique.

Les deux procédés sont donc plus proches du centre que du «noyau » de la périphérie.

La dérivation évaluative présente une autre difficulté : les dérivés évaluatifs reflètent une nette dimension pragmatique et ouvrent, en quelque sorte, le système grammatical morphologique au discours. Mais faudrait-il pour autant séparer la dérivation évaluative des autres types de dérivation?

\subsection{Lexicalisation vs. système linguistique}

Rien n'empêche pourtant qu'une unité construite par un procédé périphérique accède pleinement au statut d'unité lexicale codée, car la lexicalisation d'une unité est indépendante du mode de sa construction (CORBIN, 1992 ; FRADIN, 2003 : 220234).

Les analyses relevant de la morphologie constructionnelle peuvent être «faussées » par le phénomène de la lexicalisation. De nombreux mots issus de procédés dits «périphériques », par exemple, glouglou, RMI ou foultitude, font partie du stock « central » du lexique français : ces unités sont répertoriées dans les dictionnaires généraux et elles servent à former de nouveaux « mots », lexicalisés (glouglou, érémiste) ou non (encore) lexicalisés (érémiser, érémisation).

\footnotetext{
${ }^{16}$ Le terme de composition cachée de FRADIN $(2000,2003)$ traduit bien la nature paradoxale de ce procédé : une composition qui se cache sous les apparences d'une dérivation.
} 


\section{Connotation autonymique et morphologie constructionnelle}

\subsection{Cadrage du domaine}

Dans un deuxième temps, nous nous intéresserons au phénomène de la connotation autonymique (REY-DEBOVE, 1997: 251-291). Nous reprendrons la définition proposée au $\$ 1$ : une unité lexicale complexe, qui «dénote le monde », peut connoter sa structure morphologique. L'emploi du mot casquette dans le sens de «petit casque » (remotivation de la signification diminutive attachée à -ette), par exemple, ou d'un néologisme ludique comme Yougoslamort (remotivation abusive du segment vie et remplacement de celui-ci par son antonyme mort) se chargerait d'une connotation autonymique patente et ne passerait sans doute pas « inaperçu » aux oreilles des locuteurs francophones.

La connotation autonymique est le plus souvent associée à deux types d'unités :

- aux unités non lexicalisées ou faiblement lexicalisées construites par les procédés périphériques examinés ci-dessus ;

- aux unités néologiques et hapaxiques (cf. SABLAYROLLES, 2000) construites par les procédés constructionnels centraux, en premier lieu, par dérivation et par composition.

Les unités issues de procédés périphériques qui sont irréguliers et qui ont une faible productivité sont souvent des « mots éphémères » (ang. nonce words) créés intentionnellement à des fins affectives, ludiques ou poétiques. Il s'agit notamment des mots-valises, des mots-échos, des troncations et des mots issus de l'affixation sécrétive ou des procédés de formation argotiques. Il n'est nullement surprenant que ces unités prennent une connotation autonymique. Le traitement dictionnairique des unités lexicalisées issues de ces procédés en fait preuve : ces mots sont en règle générale assortis d'une marque d'usage ${ }^{17}$ signalant le fait qu'ils ne sont pas considérés comme non marqués, comme «neutres ».

La connotation autonymique peut également affecter les dérivés et les composés en soulignant le caractère néologique et créatif de ces unités. Un composé comme lave-aisselle (à propos des publicités pour des déodorants), construit régulièrement ( $\mathrm{V}-\mathrm{N})$, attesté (Télérama), mais non lexicalisé, n’est pas un signe «transparent» qui ne fait que dénoter son référent. L'auteur a sans doute voulu attirer l'attention de ses lecteurs sur sa création (aspect de nouveauté, analogie forte avec lave-vaisselle, charges ironique ou humoristique...).

\subsection{Connotation autonymique et discours}

L'analyse de la connotation autonymique dans le domaine de la morphologie constructionnelle se révèle complexe et dépasse largement le cadre que celle-ci s'impose ordinairement. Plusieurs facteurs d'ordres linguistique et discursif entrent en ligne de compte. Nous en effleurerons quelques-uns.

\footnotetext{
${ }^{17}$ Dans le Nouveau Petit Robert (2008), c'est la marque fam. censée signaler le registre familier. S'agit-il vraiment de la variation diastratique ?
} 


\subsubsection{Le locuteur et l'interlocuteur}

Du côté du locuteur (ou scripteur), il faut prendre en considération le caractère conscient ou non conscient de sa production, ses intentions, sa compétence lexicale et ses rapports avec l'interlocuteur.

Du côté de l'interlocuteur (ou auditeur ou lecteur), il faut trouver les réponses à la question de savoir si l'interlocuteur perçoit ou non la dimension connotative. Il faut également évaluer sa compétence lexicale et son rapport à la norme.

\subsubsection{L'échange langagier}

Ce facteur s'inscrit dans le prolongement du précédent ( $\$$ 3.2.1). Dans un premier temps, il s'agit de déterminer le caractère formel ou informel de l'échange et les relations hiérarchiques entre les participants. Ensuite, il faut évaluer la situation dans laquelle cet échange s'est déroulé. Prenons en exemple le «tollé » qu'a provoqué Ségolène Royale en employant bravitude à la place de bravoure. La connotation autonymique s'y trouve renforcée par de nombreux facteurs ${ }^{18}$ :

-il s'agissait de toute évidence d'un lapsus ${ }^{19}$ entraîné peut-être par la tournure « proverbiale $»^{20}$ de la phrase prononcée ;

- ce «barbarisme» a été proféré, dans un discours officiel, par une énarque supposée maîtriser parfaitement la langue francaise ;

- ce mot a été dit par une candidate à la présidentielle dans une situation spécifique de la campagne présidentielle de 2007.

\subsubsection{Le message}

La connotation autonymique d'un mot est souvent signalée, à l'oral, par la présence de marques suprasegmentales (notamment de l'intonation) et, à l'écrit, par les marques typographiques (l'italique ou les guillemets). Voici un exemple tiré du Canard enchaîné (du 6 mai 2009) :

«Une heure plus tôt une trentaine de ces «chauffeurs» de manifs sortaient, deux par deux, de fourgons de police stationnés boulevard Richard-Lenoir »

Les guillemets qui encadrent chauffeurs signalent un néologisme sémantique, c'est-à-dire que ce mot devrait être compris dans le sens non attesté de «celui qui excite, attise...», annoncé déjà par le titre de l'article (Ces policiers qui « chauffent » les manifs).

\footnotetext{
${ }^{18}$ Il ne faut pas perdre de vue que le suffixe - $u d e$ est un suffixe productif en français moderne qui a servi à forger des mots parfaitement lexicalisés (négritude, féminitude).

${ }^{19} \mathrm{Ni}$ le ton ni l'intonation ne signalent qu'il s'agit de l'emploi intentionnel d'un néologisme (voir $\S$ 3.2.3).

${ }^{20}$ «Comme le disent les Chinois : qui n'est pas venu sur la Grande muraille n'est pas un brave, et qui vient sur la Grande muraille conquiert la bravitude » (http://fr.wiktionary.org/wiki/bravitude)
} 


\subsubsection{La langue}

Une unité produite par un affixe (ou un procédé) peu productif (cf. DAL, 2003) est habituellement plus connotée autonymiquement qu'une unité relevant d'une règle plus productive. Un dérivé en -issime (sarkozyssime) (PERKO, à paraitre), par exemple, revêt normalement une connotation autonymique plus forte qu'un néologisme construit à partir des suffixes très productifs en -ité ('oéguité), oir(e) ( ${ }^{\circ}$ lorgnoir $)$ ou anti- ( ${ }^{\circ}$ antirépressif).

\section{Conclusion}

La dimension métalinguistique se manifeste tant au niveau systémique de la morphologie constructionnelle qu'au niveau du fonctionnement discursif des unités issues de procédés de formation des mots. Nous nous sommes attaché à démontrer que le caractère métalinguistique d'un procédé constructionnel constitue le critère discriminatif suffisant permettant de distinguer les procédés périphériques (mots-échos, mots-valises, troncations, siglaisons, affixation sécrétive) des procédés centraux (dérivation et composition). La notion de connotation autonymique, opérant au niveau discursif, pourrait remplacer des notions moins précises telles que «caractère marqué » ou «caractère intentionnel » d'un procédé constructionnel ou d'un mot complexe.

\section{BIBLIOGRAPHIE}

CORBIN, Danielle (1992), Hypothèses sur les frontières de la composition nominale, Cahiers de grammaire 17, p. 25-55.

CORBIN, Danielle (1997), Locutions, composés, unités polylexématiques : lexicalisation et mode de construction. In : M. MARTIN (éd.), La locution : entre langue et usages, Fontenay / Saint Cloud, ENS Éditions, p. 53-101

CORBIN, Danielle (2001), Préfixes et suffixes : du sens aux catégories, Journal of French Language Studies 11/1, p. 41-69.

DAL, Georgette (2003), Productivité morphologique: définitions et notions connexes, Langue française 140, p. 1-23.

Doleschal, Ursula, ThORNTON, Anna M. éds. (2000), Extragrammatical and Marginal Morphology, München, Lincom Europa.

DRESSLER, Wolfgang U. (2000), Extragrammatical vs. marginal morphology. In : Ursula, Doleschal, Anna M. THORNTON (éds.), Extragrammatical and Marginal Morphology, München, Lincom Europa, p. 1-10.

DRESSLER, Wolfgang U. (2005), Towards a natural morphology of compounding, Linguistica 45, p. 29-40.

Dressler, Wolfgang U., KAstovsky, Dieter \& PFEIFFER, Oskar E. \& RAINER, Franz (2005), Morphology and its Demarcations, Amsterdam / Philadelphia, John Benjamins.

Dressler, Wolfgang U., MaYerthaler, Willi, PANAGl, Oswald, Wurzel, Wolfgang U. (1987), Leitmotifs in natural morphology, Amsterdam / Philadelphia, John Benjamins. 
DRESSLER, Wolfgang U. \& MERLINI BARBARESI, Lavinia (1994), Morphopragmatics. Diminutives and Intesifiers in Italian, German, and other Languages, Berlin, Mouton de Gruyter.

ECO, Umberto (1988), Le signe: histoire et analyse d'un concept, Bruxelles, Éditions Labor.

FRADIN, Bernard (2000), Combining forms, blends and related phenomena. In : Ursula, Doleschal, Anna M. THORNTON (éds.), Extragrammatical and Marginal Morphology, München, Lincom Europa, p. 11-59.

FRADIN, Bernard (2003), Nouvelles approches en morphologie, Paris, PUF.

GAUDIN, Françoise $\S$ GUESPIN, Louis (2000), Initiation à la lexicologie française : de la néologie aux dictionnaires, Bruxelles, Duculot, 2000.

GrÉSILlON, Almuth (1984), La règle et le monstre, Tübingen, Max Niemeyer Verlag.

KILANI-SCHOCH, Marianne \& DRESSLER, Wolfgang U. (2005), Morphologie naturelle et flexion du verbe français, Tübingen, Gunter Narr Verlag.

MARTINET, André (1996, 1970¹), Éléments de linguistique générale, Paris, Armand Colin.

MEL'ČUK, Igor A. (1993), Cours de morphologie générale I, Montréal / Paris, Les Presses de l'Université de Montréal / CNRS Éditions.

MEL'ČUK, Igor A. (1997), Cours de morphologie générale IV, Montréal / Paris, Les Presses de l'Université de Montréal / CNRS Éditions.

MORIN, Yves-Charles (1972), The phonology of echo-words in French, Language 48/4, p. 97-108.

Nouveau Petit Robert (2008), Paris, Dictionnaire le Robert (version éléctronique).

PERKO, Gregor (à paraître), Le suffixe -issime dans le paysage dérivationnel du nom propre en français. In : Actes $d u X X V^{e}$ Congrès International de Linguistique et Philologie Romane (Innsbruck, 3-8 septembre 2007).

RADIMSKÝ, Jan (2006), Les composés italiens actuels, Paris, Cellule de Recherche en Linguistique.

ReY-Debove, Josette (1997, 1978¹), Le métalangage, Paris, Armand Colin.

SABLAYROLLES, Jean-François (1993), La double motivation de certains néologismes, Faits de langues 1, p. 223-226.

SABLAYROLlES, Jean-François (2000), La néologie en français contemporain, Paris, Honoré Champion.

\section{ABSTRACT}

The aim of this article is to analyse the connection between metalinguistic character of some world formation processes as well as some complex lexical units and their "marginal" status within most major morphological approaches. The first part of the article argues that the metalinguistic character of the process should be adopted as the only criterion for distinguishing between central and prototypical marginal word formation processes, such as echo-words, blending, clipping, abbreviations, verlan or secreted affixing. The second part applies the notion of autonymic connotation to the domain of word-formation. 